\title{
NONLINEAR OSCILLATIONS OF A COUPLED AUTOPARAMETRICAL SYSTEM WITH IDEAL AND NONIDEAL SOURCES OF POWER
}

\author{
SADO DANUTA AND KOT MACIEJ
}

Received 16 October 2005; Revised 5 June 2006; Accepted 16 July 2006

An ideal and nonideal autoparametrical system excited by DC motor with unbalanced mass is presented in this work. The system consists of the body of mass $M$ which is hung on a nonlinear spring with a nonlinear damper, and a pendulum of the length $l$ and mass $m$ mounted to the body of mass $M$. It is assumed that the motion of the pendulum is damped by nonlinear resistive forces. Vibrations of both models (ideal and nonideal) are researched. Solutions for the system response are presented for specific values of the parameters of system and the energy transfer between modes of vibrations is studied. Next excited vibrations for both models have been examined analytically and numerically. Except different kinds of periodic vibrations, there may also appear chaotic vibrations.

Copyright (C 2006 S. Danuta and K. Maciej. This is an open access article distributed under the Creative Commons Attribution License, which permits unrestricted use, distribution, and reproduction in any medium, provided the original work is properly cited.

\section{Introduction}

In this work, the nonlinear dynamics of a two-degree-of-freedom autoparametric system excited by DC motor with unbalanced mass as model ideal or nonideal is studied. Dynamical systems with elements of the mathematical or physical pendulum type have important applications. If the pendulum is attached to the body suspended on the flexible element, in this system may occur the autoparametric excitation as a result of inertial coupling. The internal resonance of a parametric type may occur in the systems of this type. The influence of different types of nonlinearities on conditions of internal or external resonances has fundamental meaning. Depending on the excitation is influenced or not by the response of the system, the vibrating system may be called ideal or nonideal. When a forcing function is independent of the system it acts on, then the forcing function is called ideal. The ideal problems are the traditional ones. Formally, the excitation is expressed as a pure function of time, for example, by a sinusoidal excitation. In this case, the excitation is independent of the system response. On the other hand, a forcing function depending on the response of the system is said to be nonideal. When we use a nonideal source of power instead of an ideal one, the excitation should be presented as a 
function, which depends on the response of the system. In this case, the nonideal source of power cannot be expressed as a pure function of time, but as an equation that relates the source of energy to the system. Then the energy supplying effect is described by another equation. The first detailed study on the nonideal vibrating systems is presented by Kononenko [12]. He obtained satisfactory results by the comparison of the experimental analysis and approximated methods. After this publication, the problem of nonideal vibrating systems was investigated by a number of authors. The nonideal problem was presented by Evan-Ivanowski [9] or Nayfeh and Mook [15]. These authors showed that sometimes dynamical coupling between energy sources and structural response that had not to be ignored in real engineering problems. A complete review on different theories on nonideal vibrating systems was discussed and presented by Balthazar et al. [5]. Nonideal models were researched by Krasnopol'skaya and Shvets [13]. These authors analyzed a physical model consisting of a crank mechanism coupled to the support point of a simple pendulum. Belato et al. [6] studied nonideal similar system consisting of a pendulum whose support point is vibrated along a horizontal guide by a two-bar linkage driven by a limited power of DC motor. Vibrations of ideal and nonideal parametrical and selfexcited models were described by Půst and Szöllös [16] and Warmiński et al. [27]. Cavalca et al. [8] studied a nonlinear model for the Laval rotor with an unlimited power source. Model of flexible slewing structures with DC motor was investigated by Fenili et al. [10]. Possibilities of existence of regular and irregular motions in nonideal vibrating problems were shown for example by Warmiński [26], Belato et al. [7], or Tsuchida et al. [25].

An autoparametrical system with ideal and nonideal excitations is investigated in this paper. This kind of system with harmonic excitation called ideal is well known in the literature (Bajaj and Johnson [2], Bajaj and Tousi [3], Bajaj et al. [1], Hatwal et al. [11], Sado [17-19]). The present paper is a continuation of the authors' previous works where different types of autoparametric systems with inertial coupling were investigated. Near the internal and external resonance regions, except different kinds of periodic vibration, the chaotic vibration was observed for a two-degree-of-freedom beam-pendulum system (Sado [17-20]), for double pendulum system (Sado and Gajos [22]), or for system with spring pendulum (Sado [21]). This phenomenon was served also for nonideal model (Sado and Kot $[23,24]$ ). In present paper, the analysis is carried out in the wide aspects of the influence of different kinds of nonlinearities. Taking into account geometric, elastic, and damping nonlinearities, the influence of motor's speed on the behavior of the system is discussed. The equations of motion are solved numerically what permits to enrich the investigations and to examine not only small and steady state oscillations but also large amplitude oscillations in transient states. The present paper shows that in this type of nonideal system, one mode of vibrations may excite or damp another mode, and near the resonance regions, except multiperiodic and quasiperiodic vibrations, also chaotic motions there may appear. For characterizing an irregular chaotic response in this work, the bifurcation diagrams for different damping parameters, and time histories, power spectral densities (using FFT), Poincaré maps, and maximal exponents of Lyapunov are constructed. These descriptors are available to observe chaos, and to better understand behavior of the system (Moon [14], Baker and Gollub [4]). When a bifurcation diagram is plotted, several phenomena can be observed: existence of a simple attractor with low 


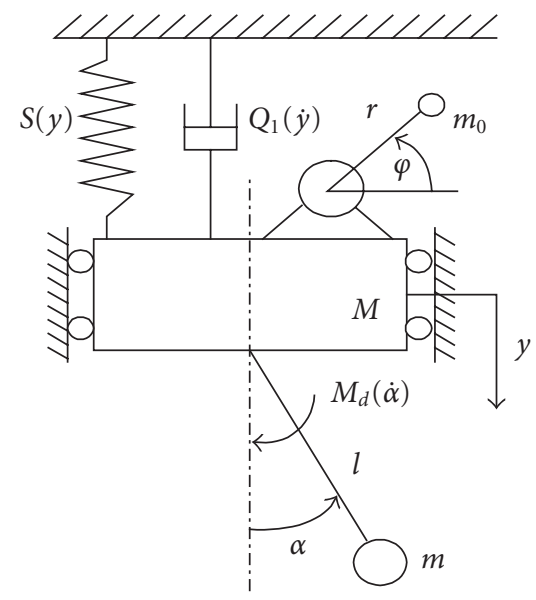

Figure 2.1. Schematic diagram of the system.

period, or existence of a chaotic attractor and various bifurcations. All these effects are verified in the phase space. Nonperiodic attractors are traced by solving an initial value problem. Fast Fourier transform is used to obtain a power spectrum which delivers information about the nature of the investigated periodic and nonperiodic attractors. The maximum Lyapunov exponents are calculated in order to characterize the chaotic orbits.

\section{System description and equation of motion}

The investigated system is shown in Figure 2.1. The system consists of a pendulum and a body of mass $M$ suspended on the flexible element characterized by nonlinear elasticity of rigidity $k$ and nonlinear viscous damping. A pendulum with a weightless rod of length $l$ and a lumped mass $m$ is mounted to the body of mass $M$. It is assumed that a nonlinear viscous damping force applied in the hinge opposes the motion of the pendulum. The body of mass $M$ is subjected to excitation by an electric motor with unbalanced mass $m_{0}$ (modeled as ideal or nonideal system). It was assumed that the elasticity force is $S(y)=$ $k_{1}\left(y+y_{\mathrm{st}}\right)+k_{2}\left(y+y_{\mathrm{st}}\right)^{3}$, where $y_{\mathrm{st}}$ is the static vertical displacement and $k_{1}$ and $k_{2}$ are constant coefficients of elasticity. Also it was assumed that the damping force acting on the body $M$ is $Q_{1}(\dot{y})=C_{1} \dot{y}+C_{3} \dot{y}^{3}$, while the resistive moment acting on the pendulum is $M_{d}(\dot{\alpha})=C_{2} \dot{\alpha}+C_{4} \dot{\alpha}^{3}$, where $C_{1}, C_{2}, C_{3}, C_{4}$ are constant coefficients of damping.

Vibrations are researched around static point of balance and equations of motion are derived by applying Lagrange's equations. This system is considered as system ideal or nonideal.

2.1. Ideal system. The system presented in Figure 2.1 has two degrees of freedom. In this case, excitation is expressed as a pure function of time represented by vertical component of inertial force $F=m_{0} v^{2} r \sin (v t)$. The generalized coordinates are: the vertical displacement $y$ of main mass $M$, the angular displacement $\alpha$ of a pendulum measured from its lower position. The static vertical displacement $y_{\text {st }}$ can be found from 
$4 \quad$ Ideal and nonideal autoparametrical system

$(M+m) g=k_{1} y_{\mathrm{st}}+k_{2} y_{\mathrm{st}}^{3}$, where $g$ is acceleration of gravity. The kinetic energy $T$ and the potential energy $V$ of the system are

$$
\begin{gathered}
T=\frac{1}{2}(M+m) \dot{y}^{2}-m l \dot{y} \dot{\alpha} \sin \alpha+\frac{1}{2} m l^{2} \dot{\alpha}^{2}, \\
V=\frac{1}{2} k_{1}\left(y+y_{\text {st }}\right)^{2}+\frac{1}{4} k_{2}\left(y+y_{\text {st }}\right)^{4}-(M+m) g y+m g l(1-\cos \alpha)
\end{gathered}
$$

and then the equations of motion of the system take the following form:

$$
\begin{gathered}
(M+m) \ddot{y}-m l \ddot{\alpha} \sin \alpha-m l \dot{\alpha}^{2} \cos \alpha+k_{1}\left(y+y_{\mathrm{st}}\right) \\
+k_{2}\left(y+y_{\mathrm{st}}\right)^{3}-(M+m) g=-C_{1} \dot{y}-C_{3} \dot{y}^{3}-m_{0} v^{2} r \sin v t \\
m l^{2} \ddot{\alpha}-m l \ddot{y} \sin \alpha+m g l \sin \alpha=-C_{2} \dot{\alpha}-C_{4} \dot{\alpha}^{3} .
\end{gathered}
$$

After introduction, dimensionless time $\tau=\omega_{1} t$ and the following dimensionless coefficients are

$$
\begin{gathered}
y_{1}=\frac{y}{y_{\mathrm{st} L}} ; \quad y_{1 \mathrm{st}}=\frac{y_{\mathrm{st}}}{y_{\mathrm{st} L}} ; \quad \omega_{1}^{2}=\frac{k_{1}}{M+m} ; \quad \omega_{2}^{2}=\frac{g}{l} ; \quad \beta=\frac{\omega_{2}}{\omega_{1}} ; \quad \mu=\frac{\nu}{\omega_{1}} ; \\
a_{1}=\frac{m_{0} r}{(M+m) y_{\mathrm{st} L}} ; \quad a_{2}=\frac{m}{M+m} ; \quad b_{1}=\frac{k_{2} l^{2}}{k_{1}} ; \quad \gamma_{1}=\frac{C_{1}}{(M+m) \omega_{1}} ; \\
\gamma_{2}=\frac{C_{2}}{m l^{2} \omega_{1}} ; \quad \gamma_{3}=\frac{C_{3} l^{2} \omega_{1}}{M+m} ; \quad \gamma_{4}=\frac{C_{4} \omega_{1}}{m l^{2}} ;
\end{gathered}
$$

equations of motion can be written in the form

$$
\begin{aligned}
& \ddot{y}_{1}=\left[a_{2} \frac{1}{\beta^{2}} \dot{a}_{1} \cos \alpha-y_{1}-b_{1} \beta^{4}\left(y_{1}^{3}+3 y_{1}^{2} y_{1 \mathrm{st}}+3 y_{1} y_{1 \mathrm{st}}^{2}\right)-\gamma_{1} \dot{y}_{1}-\gamma_{3} \dot{y}_{1}^{3}\right. \\
& \left.+a_{1} \mu^{2} \sin \mu \tau+a_{2} \frac{1}{\beta^{2}}\left(-\beta^{2} \sin \alpha-\gamma_{2} \dot{\alpha}-\gamma_{4} \dot{\alpha}^{3}\right) \sin \alpha\right] \times \frac{1}{\left(1-a_{2} \sin ^{2} \alpha\right)}, \\
& \ddot{\alpha}=\left\{-\beta^{2} \sin \alpha-\gamma_{2} \dot{\alpha}-\gamma_{4} \dot{\alpha}^{3}+\beta^{2}\left[a_{2} \frac{1}{\beta^{2}} \dot{\alpha}^{2} \cos \alpha-y_{1}-b_{1} \beta^{4}\left(y_{1}^{3}+3 y_{1}^{2} y_{\mathrm{st} 1}+3 y_{1} y_{\mathrm{st} 1}^{2}\right)\right.\right. \\
& \left.\left.-\gamma_{1} \dot{y}_{1}-\gamma_{3} \dot{y}_{1}^{3}+a_{1} \mu^{2} \sin \mu \tau\right] \sin \alpha\right\} \times \frac{1}{\left(1-a_{2} \sin ^{2} \alpha\right)} .
\end{aligned}
$$

2.2. Nonideal system. In this case, the DC motor is considered as a nonideal source of power (the power supply is limited). The nonideal source of power adds one degree of 
freedom, it means that the system has three degrees of freedom. The generalized coordinates are: the vertical displacement $y$ of main mass $M$, the angular displacement $\alpha$ of a pendulum measured from vertical line, and coordinate $\varphi$ which describes angular displacement of the unbalanced mass $m_{0}$ measured from horizontal line. The static vertical displacement $y_{\mathrm{st}}$ in this case can be found from $\left(M+m+m_{0}\right) g=k_{1} y_{\mathrm{st}}+k_{2} y_{\mathrm{st}}^{3}$.

The kinetic energy $T$ and the potential energy $V$ of the system are

$$
\begin{gathered}
T=\frac{1}{2}\left(I_{0}+m_{0} r^{2}\right) \dot{\varphi}^{2}+\frac{1}{2}\left(M+m+m_{0}\right) \dot{y}^{2}+\frac{1}{2} m l^{2} \dot{\alpha}^{2}-m_{0} r \dot{y} \dot{\varphi} \cos \varphi-m l \dot{y} \dot{\alpha} \sin \alpha, \\
V=\frac{1}{2} k_{1}\left(y+y_{\mathrm{st}}\right)^{2}+\frac{1}{4} k_{2}\left(y+y_{\mathrm{st}}\right)^{4}-M g y+m_{0} g(r \sin \varphi-y)+m g(l-l \cos \alpha-y) .
\end{gathered}
$$

The equations of motion of the system take the following form:

$$
\begin{gathered}
\left(M+m+m_{0}\right) \ddot{y}-m l \dot{\alpha}^{2} \cos \alpha-m l \ddot{\alpha} \sin \alpha+m_{0} r \dot{\varphi}^{2} \sin \varphi-m_{0} r \ddot{\varphi} \cos \varphi \\
+k_{1} y+k_{2} y^{3}+3 k_{2} y^{2} y_{\mathrm{st}}+3 k_{2} y y_{\mathrm{st}}^{2}+C_{1} \dot{y}+C_{3} \dot{y}^{3}=0, \\
m l^{2} \ddot{\alpha}-m l \ddot{y} \sin \alpha+m g l \sin \alpha+C_{2} \dot{\alpha}+C_{4} \dot{\alpha}^{3}=0, \\
\left(I+m_{0} r^{2}\right) \ddot{\varphi}-m_{0} \ddot{y} r \cos \varphi+m_{0} g r \cos \varphi=L(\dot{\varphi})-H(\dot{\varphi}),
\end{gathered}
$$

where $L(\dot{\varphi})$ is a driving torque of DC motor and $H(\dot{\varphi})$ is a resistance torque.

The following dimensionless definitions are introduced in this model:

$$
\begin{gathered}
y_{1}=\frac{y}{y_{\mathrm{st} L}} ; \quad y_{1 \mathrm{st}}=\frac{y_{\mathrm{st}}}{y_{\mathrm{st} L}} ; \quad \omega_{1}^{2}=\frac{k_{1}}{M+m+m_{0}} ; \quad \omega_{2}^{2}=\frac{g}{l} ; \quad \beta=\frac{\omega_{2}}{\omega_{1}} ; \\
a_{1}=\frac{m_{0} r}{\left(M+m+m_{0}\right) y_{\mathrm{st} L}} ; \quad a_{2}=\frac{m}{M+m+m_{0}} ; \quad b_{1}=\frac{k_{2} l_{1}^{2}}{k_{1}} ; \\
\gamma_{1}=\frac{C_{1}}{\left(M+m+m_{0}\right) \omega_{1}} ; \quad \gamma_{2}=\frac{C_{2}}{m^{2} \omega_{1}} ; \quad \gamma_{3}=\frac{C_{3} l^{2} \omega_{1}}{M+m+m_{0}} ; \\
\gamma_{4}=\frac{C_{4} \omega_{1}}{m^{2}} ; \quad q=\frac{m_{0} r y_{\mathrm{st} L}}{I+m_{0} r^{2}} \quad G_{1}(\dot{\varphi})=\frac{L(\dot{\varphi})-H(\dot{\varphi})}{\left(I+m_{0} r^{2}\right) \omega_{1}^{2}} ; \quad \tau=\omega_{1} t
\end{gathered}
$$

where $G_{1}(\dot{\varphi})=u_{1}-u_{2} \dot{\varphi}$, where the parameter $u_{1}$ is related to the voltage, and $u_{2}$ is constant parameter for each model of motor considered. The voltage (parameter $u_{1}$ ) is treated as a control parameter. 
$6 \quad$ Ideal and nonideal autoparametrical system

After transformations, equations of motion can be written in the form:

$$
\begin{aligned}
\ddot{y}_{1}= & {\left[a_{1} \dot{\varphi}^{2} \sin \varphi-\frac{a_{2}}{\beta^{2}} \dot{\alpha}^{2} \cos \alpha+y_{1}+\beta^{4}\left(b_{1} y_{1}^{3}+3 b_{1} y_{\mathrm{st} 1} y_{1}^{2}+3 b_{1} y_{\mathrm{st} 1_{1}}^{2}+\gamma_{3} \dot{y}_{1}^{3}\right)+\gamma_{1} \dot{y}_{1}\right.} \\
& \left.+\left(\sin \alpha+\frac{1}{\beta^{2}} \gamma_{2} \dot{\alpha}+\frac{1}{\beta^{2}} \gamma_{4} \dot{\alpha}^{3}\right) a_{2} \sin \alpha-\left(u_{1}-u_{2} \dot{\varphi} o s \varphi\right) a_{1} \cos \varphi\right] \\
& \times \frac{1}{\left(a_{2} \sin ^{2} \alpha+a_{1} q \cos ^{2} \varphi-1\right)} \\
\ddot{\alpha}=[ & \left(a_{1} \beta^{2} \dot{\varphi}^{2} \sin \varphi-a_{2} \dot{\alpha}^{2} \cos \alpha+\beta^{2} y_{1}+\beta^{6} b_{1} y_{1}^{3}\right. \\
& \left.+3 \beta^{6} b_{1}\left(y_{\mathrm{st} 1} y_{1}^{2}+y_{\mathrm{st} 1}^{2} y_{1}\right)+\beta^{2} \gamma_{1} \dot{y}_{1}+\beta^{6} \gamma_{3} y_{1}^{3}\right) \sin \alpha \\
& \left.-\left(q a_{1} \cos { }^{2} \varphi-1\right)\left(\beta^{2} \sin \alpha+\gamma_{2} \dot{\alpha}+\gamma_{4} \dot{\alpha}^{3}\right)-a_{1} \beta^{2} \cos \varphi \cos \alpha\left(u_{1}-u_{2} \dot{\varphi}-q \cos \varphi\right)\right] \\
\times & \frac{1}{\left(a_{2} \sin ^{2} \alpha+a_{1} q \cos ^{2} \varphi-1\right)} \\
\ddot{\varphi}= & \left(a_{1} \dot{\varphi}^{2} \sin ^{2}-\frac{a_{2}}{\beta^{2}} \dot{\alpha}^{2} \cos \alpha+y_{1}+\beta^{4} b_{1} y_{1}^{3}+\gamma_{1} \dot{y}_{1}+3 \beta^{4} b_{1} y_{\mathrm{st} 1} y_{1}^{2}\right. \\
& \left.+3 \beta^{4} b_{1} y_{\mathrm{st} 1}^{2} y_{1}+\beta^{4} \gamma_{3} \dot{y}_{1}^{3}\right) q \cos \varphi+a_{2} q \cos \varphi \sin \alpha\left(\sin \alpha+\frac{1}{\beta^{2}} y_{2} \dot{\alpha}+\frac{1}{\beta^{2}} \gamma_{4} \dot{\alpha}^{3}\right) \\
& \left.+\left(u_{1}-u_{2} \dot{\varphi}-q \cos \varphi\right)\left(a_{2} \sin ^{2} \alpha-1\right)\right] \\
\times & \frac{1}{\left(a_{2} \sin ^{2} \alpha+a_{1} q \cos ^{2} \varphi-1\right)} .
\end{aligned}
$$

The nonideal problem is described by a set of autonomous equations. Time does not appear in (2.9).

\section{Analytical solution}

The method of multiple scales is used to obtain approximate solutions of (2.4) and (2.5).

By introduction, formal small parameter $\varepsilon$ coefficients in (2.4) and (2.5) are defined as

$$
a_{1}=\varepsilon^{2} \bar{a}_{1}, \quad b_{1}=\varepsilon \bar{b}_{1}, \quad \gamma_{1}=\varepsilon \bar{\gamma}_{1}, \quad \gamma_{2}=\varepsilon \bar{\gamma}_{2}, \quad \gamma_{3}=\varepsilon \bar{\gamma}_{3}, \quad \gamma_{4}=\varepsilon \bar{\gamma}_{4} .
$$

Following the method of multiple scales (Nayfeh, 1979), the solutions are expressed in the form

$$
\begin{aligned}
& y\left(T_{0}, T_{1}, T_{2}, \ldots, \varepsilon\right) \\
& =\varepsilon y_{0}\left(T_{0}, T_{1}, T_{2} \cdots\right)+\varepsilon^{2} y_{1}\left(T_{0}, T_{1}, T_{2} \cdots\right)+\varepsilon^{3} y_{2}\left(T_{0}, T_{1}, T_{2} \cdots\right)+\cdots, \\
& \alpha\left(T_{0}, T_{1}, T_{2}, \ldots, \varepsilon\right) \\
& =\varepsilon \alpha_{0}\left(T_{0}, T_{1}, T_{2} \cdots\right)+\varepsilon^{2} \alpha_{1}\left(T_{0}, T_{1}, T_{2} \cdots\right)+\varepsilon^{3} \alpha_{2}\left(T_{0}, T_{1}, T_{2} \cdots\right)+\cdots,
\end{aligned}
$$


where $T_{0}=\tau, T_{1}=\varepsilon \tau, T_{2}=\varepsilon^{2} \tau, \ldots$ and next governing equations in different perturbation orders are the following:

for $\varepsilon^{1}$ :

$$
\begin{gathered}
\frac{\partial^{2} y_{0}}{\partial T_{0}^{2}}+y_{0}=0, \\
\frac{\partial^{2} \alpha_{0}}{\partial T_{0}^{2}}+\beta^{2} \alpha_{0}=0,
\end{gathered}
$$

for $\varepsilon^{2}$ :

$$
\begin{gathered}
\frac{\partial^{2} y_{1}}{\partial T_{0}^{2}}+y_{1}=-2 \frac{\partial^{2} y_{0}}{\partial T_{0} \partial T_{1}}+\frac{1}{\beta^{2}} a_{2} \frac{\partial^{2} \alpha_{0}}{\partial T_{0}^{2}} \alpha_{0}+\frac{1}{\beta^{2}} a_{2}\left(\frac{\partial \alpha_{0}}{\partial T_{0}}\right)^{2} \\
-3 b_{1} \beta^{4} y_{0} y_{\mathrm{st} 1}^{2}-\gamma_{1} \frac{\partial y_{0}}{\partial T_{0}}-a_{1} \mu^{2} \sin \mu T_{0} \\
\frac{\partial^{2} \alpha_{1}}{\partial T_{0}^{2}}+\beta^{2} \alpha_{1}=-2 \frac{\partial^{2} \alpha_{0}}{\partial T_{0} \partial T_{1}}+\beta^{2} \alpha_{0} \frac{\partial^{2} y_{0}}{\partial T_{0}^{2}}-\gamma_{2} \frac{\partial \alpha_{0}}{\partial T_{0}}
\end{gathered}
$$

for $\varepsilon^{3}$ :

$$
\begin{aligned}
\frac{\partial^{2} y_{2}}{\partial T_{0}^{2}}+y_{2}= & -2 \frac{\partial^{2} y_{1}}{\partial T_{0} \partial T_{1}}-2 \frac{\partial^{2} y_{0}}{\partial T_{0} \partial T_{2}}-\frac{\partial^{2} y_{0}}{\partial T_{1}^{2}}+\frac{1}{\beta^{2}} a_{2} \frac{\partial^{2} \alpha_{1}}{\partial T_{0}^{2}} \alpha_{0} \\
& +\frac{1}{\beta^{2}} a_{2} \frac{\partial^{2} \alpha_{0}}{\partial T_{0} \partial T_{1}} \alpha_{0}+\frac{1}{\beta^{2}} a_{2} \frac{\partial^{2} \alpha_{0}}{\partial T_{0}^{2}} \alpha_{1}+\frac{1}{\beta^{2}} a_{2}\left(\frac{\partial \alpha_{0}}{\partial T_{0}} \frac{\partial \alpha_{1}}{\partial T_{0}}-\frac{\partial \alpha_{0}}{\partial T_{0}} \frac{\partial \alpha_{0}}{\partial T_{1}}\right) \\
& -3 b_{1} \beta^{4} y_{0}^{2} y_{\mathrm{st} 1}-3 b_{1} \beta^{4} y_{1} y_{\mathrm{st} 1}^{2}-\gamma_{1} \frac{\partial y_{1}}{\partial T_{0}}-\gamma_{1} \frac{\partial y_{0}}{\partial T_{1}} .
\end{aligned}
$$

The solutions of (3.3) can be written as

$$
\begin{gathered}
y_{0}\left(T_{0}, T_{1}, T_{2}\right)=A\left(T_{1}, T_{2}\right) e^{i T_{0}}+\bar{A}\left(T_{1}, T_{2}\right) e^{-i T_{0}}, \\
\alpha_{0}\left(T_{0}, T_{1}, T_{2}\right)=B\left(T_{1}, T_{2}\right) e^{i \beta T_{0}}+\bar{B}\left(T_{1}, T_{2}\right) e^{-i \beta T_{0}} .
\end{gathered}
$$

Substituting (3.6) into (3.4) leads to

$$
\begin{aligned}
\frac{\partial^{2} y_{1}}{\partial T_{0}^{2}}+y_{1}= & -2 i \frac{\partial A}{\partial T_{1}} e^{i T_{0}}-2 \frac{1}{\beta^{2}} a_{2} B^{2} \beta^{2} e^{2 i \beta T_{0}}-3 b_{1} \beta^{4} y_{\text {st } 1}^{2} A e^{i T_{0}} \\
& -\gamma_{1} i A e^{i T_{0}}-\frac{1}{2 i} a_{1} \mu^{2} e^{i \mu T_{0}}+\text { c. c. }, \\
\frac{\partial^{2} \alpha_{1}}{\partial T_{0}^{2}}+\beta^{2} \alpha_{1}= & -2 i \beta \frac{\partial B}{\partial T_{1}} e^{i \beta T_{0}}+\beta^{2}\left(-A B e^{i T_{0}(1+\beta)}-A \bar{B} e^{i T_{0}(1-\beta)}\right) \\
& -i \gamma_{2} B \beta e^{i \beta T_{0}}+\text { c. c. },
\end{aligned}
$$

where c.c. represents the complex conjugate to the terms presented in (3.7). 
8 Ideal and nonideal autoparametrical system

Thus, to eliminate terms that produce secular terms, we need to distinguish also the following cases:

$$
\begin{gathered}
2 \beta=1, \\
\mu=1, \\
1-\beta=\beta .
\end{gathered}
$$

For $\beta=0.5$ is internal resonance and for $\mu=1$ is external resonance, so we assume that $\mu=1+\varepsilon \sigma_{1}$ and $2 \beta=1-\varepsilon \sigma_{2}$, where $\sigma_{1} i \sigma_{2}$ are detuning parameters.

Secular terms are eliminated if

$$
\begin{gathered}
-2 i \frac{\partial A}{\partial T_{1}} e^{i T_{0}}-2 B^{2} a_{2} e^{-i \sigma_{2} T_{1}} e^{i T_{0}}-3 b_{1} \beta^{4} y_{\mathrm{st} 1}^{2} A e^{i T_{0}}-\gamma_{1} A i e^{i T_{0}}+\frac{1}{2 i} a_{1} \mu^{2} e^{i \sigma_{1} T_{1}} e^{i T_{0}}=0 \\
-2 i \frac{\partial B}{\partial T_{1}} e^{i \beta T_{0}}-\beta A \bar{B} e^{i \sigma_{2} T_{1}} e^{i \beta T_{0}}-\gamma_{2} i B e^{i \beta T_{0}}=0
\end{gathered}
$$

Letting $A=(1 / 2) a e^{i \phi_{1}}, \bar{A}=(1 / 2) a e^{-i \phi_{1}}, B=(1 / 2) b e^{i \phi_{2}}, \bar{B}=(1 / 2) b e^{-i \phi_{2}}$ and separating real and imaginary parts, we have

$$
\begin{gathered}
2 a^{\prime}=a_{2} b^{2} \sin \theta_{2}-a \gamma_{1}+a_{1} \mu^{2} \cos \theta_{1}, \\
2 a \phi_{1}^{\prime}=a_{2} b^{2} \cos \theta_{2}+3 b_{1} \beta^{4} y_{\text {st } 1}^{2} a+a_{1} \mu^{2} \sin \theta_{1}, \\
b^{\prime}=\frac{1}{4} \beta a b \sin \theta_{2}+\frac{1}{2} \gamma_{2} b, \\
b \phi_{2}^{\prime}=\frac{1}{4} \beta a b \cos \theta_{2},
\end{gathered}
$$

where $\theta_{1}=\sigma_{1} T_{1}-\phi_{1}, \theta_{2}=\phi_{1}-2 \phi_{2}+\sigma_{2} T_{1}$.

For a steady state $a^{\prime}=0, \theta_{1}^{\prime}=0, b^{\prime}=0, \theta_{2}^{\prime}=0$, and thus

$$
\begin{gathered}
-a_{2} b^{2} \sin \theta_{2}+a \gamma_{1}-a_{1} \mu^{2} \cos \theta_{1}=0, \\
2 a \sigma_{1}-a_{2} b^{2} \cos \theta_{2}-3 b_{1} \beta^{4} y_{\text {st } 1}^{2} a-a_{1} \mu^{2} \sin \theta_{1}=0, \\
2\left(\sigma_{1}+\sigma_{2}\right) b-\beta a b \cos \theta_{2}=0, \\
-\beta a b \sin \theta_{2}-2 \gamma_{2} b=0 .
\end{gathered}
$$

The following two cases of solutions of (3.11) are possible:

(1) $b=0$ and $a \neq 0$, so

$$
a=\frac{a_{1} \mu^{2}}{\sqrt{\gamma_{1}^{2}+\left(2 \sigma_{1}-3 b_{1} \beta^{4} y_{\mathrm{st} 1}^{2}\right)^{2}}}, \quad \theta_{1}=\operatorname{arctg} \frac{3 b_{1} \beta^{4} y_{\mathrm{st} 1}^{2}-2 \sigma_{1}}{\gamma_{1}}
$$

and $\theta_{2}$ is arbitrary.

In this case, the pendulum does not vibrate. 
(2) $a \neq 0$ and $b \neq 0$, so

$$
\begin{gathered}
a=\frac{2 \sqrt{\left(\sigma_{1}+\sigma_{2}\right)^{2}+\gamma_{2}^{2}}}{\beta}, \quad \theta_{2}=\operatorname{arctg}\left(-\frac{\gamma_{2}}{\left(\sigma_{1}-\sigma_{2}\right)}\right), \\
b^{2}=\frac{\left\{2\left[\gamma_{1} \gamma_{2}-\left(\sigma_{1}+\sigma_{2}\right) \Lambda\right] / \beta \pm \sqrt{4\left[\gamma_{1} \gamma_{2}-\left(\sigma_{1}+\sigma_{2}\right) \Lambda\right]^{2} / \beta^{2}-\left[a^{2}\left(\gamma_{1}^{2}+\Lambda^{2}\right)-a_{1}^{2} \mu^{4}\right]}\right\}}{a_{2}}, \\
\theta_{1}=\operatorname{arctg}\left(\frac{a^{2} \beta \Lambda-2 a_{2} b^{2}\left(\sigma_{1}+\sigma_{2}\right)}{\beta a^{2} \gamma_{1}+2 a_{2} b^{2} \gamma_{2}}\right),
\end{gathered}
$$

where $\Lambda=2 \sigma_{1}-3 b_{1} \beta^{4} y_{\text {st } 1}^{2}$.

Both the pendulum and the oscillator vibrate.

\section{Numerical results}

Equations of motion of ideal and nonideal systems are solved numerically for autoparametric resonance conditions defined in Section 3. The calculations are carried out for different values of parameters of the system. The main internal resonance occurs for parameter $\beta$ near 0.5 while the external resonance occurs for parameter $\mu$ near 1 .

4.1. Free vibrations of ideal system. Equations (2.5) were solved numerically for parameter $a_{1}=0$. The influence of damping parameters on behavior of the system has been investigated. Exemplary results obtained for free vibrations are presented in Figures 4.1 and 4.2. As one can see, increasing value of damping coefficient $\gamma_{1}$ (put on body of mass $M)$ causes reduction of amplitudes $y_{1}$ and $\alpha$ and extends the time of vibrations transfer between degrees of freedom. The same effect one can see while increasing the value of damping coefficient $\gamma_{2}$ put on the pendulum (Figure 4.2).

Next is analyzed the influence of the spring nonlinearity on the energy transfer. The internal resonant curves for initial conditions put on pendulum and for various nonlinear coefficients $b_{1}$ are presented in Figure 4.3. As it can be seen from the presented diagram for larger values of $b_{1}$, we can observe new internal resonance areas (for $b_{1}=0.8$ near the resonance ratio $\beta=1.7$ and for $b_{1}=1$ near the resonance ratio $\beta=1.6$ ).

4.2. Excited vibrations of ideal system. The influence of damping acting on the body $M$ and on the pendulum excited by an external harmonic force is investigated. Exemplary results are presented in Figures 4.4 and 4.5. We can see that even small damping strongly affects the energy transfer between both vibration modes. Exemplary resonant curves (amplitude versus excitation frequency) under the internal resonance conditions are presented in Figure 4.6.

Near the internal and external resonances depending on a selection of physical system parameters, the amplitudes of vibrations of both coupled bodies may be related differently. The system presents some interesting nonlinear phenomena. Motions $y_{1}$ and $\alpha$ are harmonic, multiperiodic or quasiperiodic, but sometimes the motions of the body 

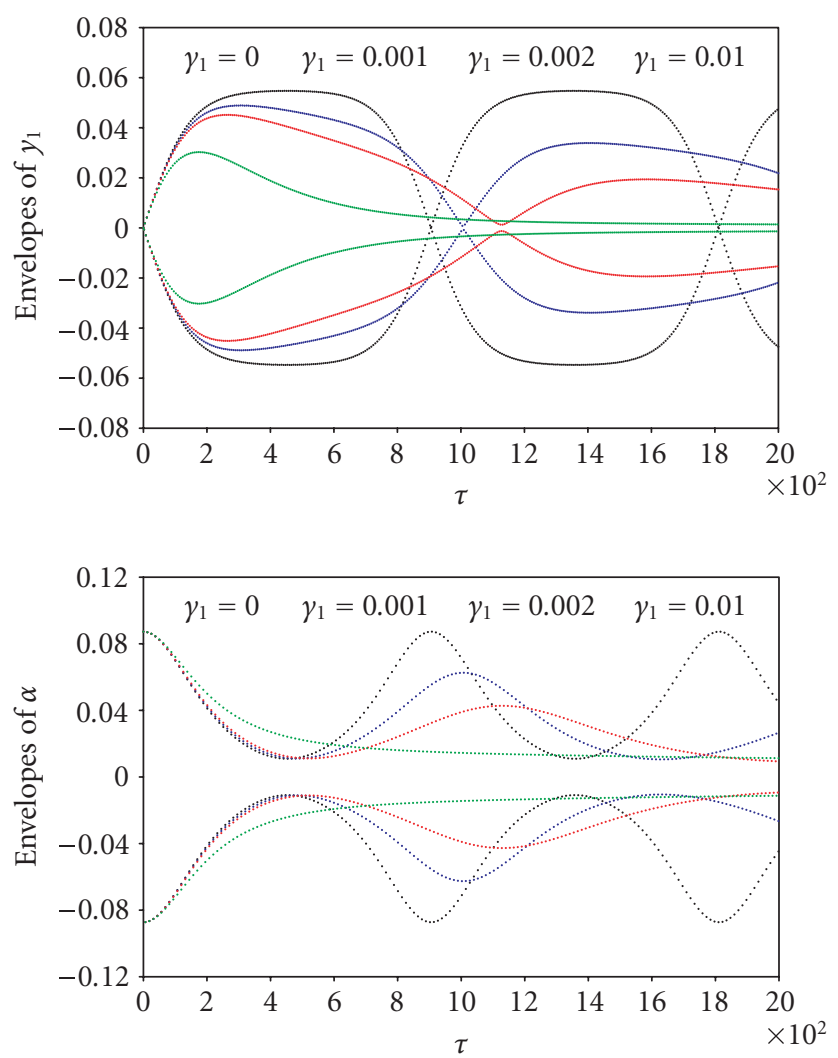

Figure 4.1. Influence of damping $\gamma_{1}$ on $y_{1}$ and $\alpha$ for $\beta=0.5, a_{1}=0, a_{2}=0.1, \gamma_{2}=\gamma_{3}=\gamma_{4}=0, b_{1}=0$.

of mass $M$ and of the pendulum are chaotic. For characterizing, an irregular chaotic response forms a transition zone between one and another type of regular steady resonant motion, in the present paper, the bifurcation diagrams are constructed (Figures 4.7 and 4.8). We can observe existence of periodic or chaotic motions. These phenomena have to be verified, so next we observe Poincarè maps and Lyapunov exponents. Exemplary results are presented in Figures 4.9 and 4.10. As can be seen from these figures for presented values of bifurcation parameter $a_{1}=0.007$, the Poincarè maps trace the strange attractors and the largest Lyapunov exponents are positive, so presented motions are chaotic.

4.3. Excited vibrations of nonideal system. The equations of motion have been solved numerically. Calculations have been done for different values of the system parameters and for the following parameters of DC motor: $u_{1}=0.2 \div 4, u_{2}=1.5$, where $u_{1}$ is a control parameter which depends on voltage and parameter $u_{2}$ depends on the type of energy source. Calculations incorporate the following initial conditions: $\dot{\varphi}(0)=1, \alpha(0)=0.005^{\circ}$, $\varphi(0)=y(0)=\dot{y}(0)=\dot{\alpha}(0)=0$ and the parameters $\beta=0.5, q=0.2$. Exemplary results of time histories and their envelopes in the longer time corresponding to displacements $y_{1}$ 

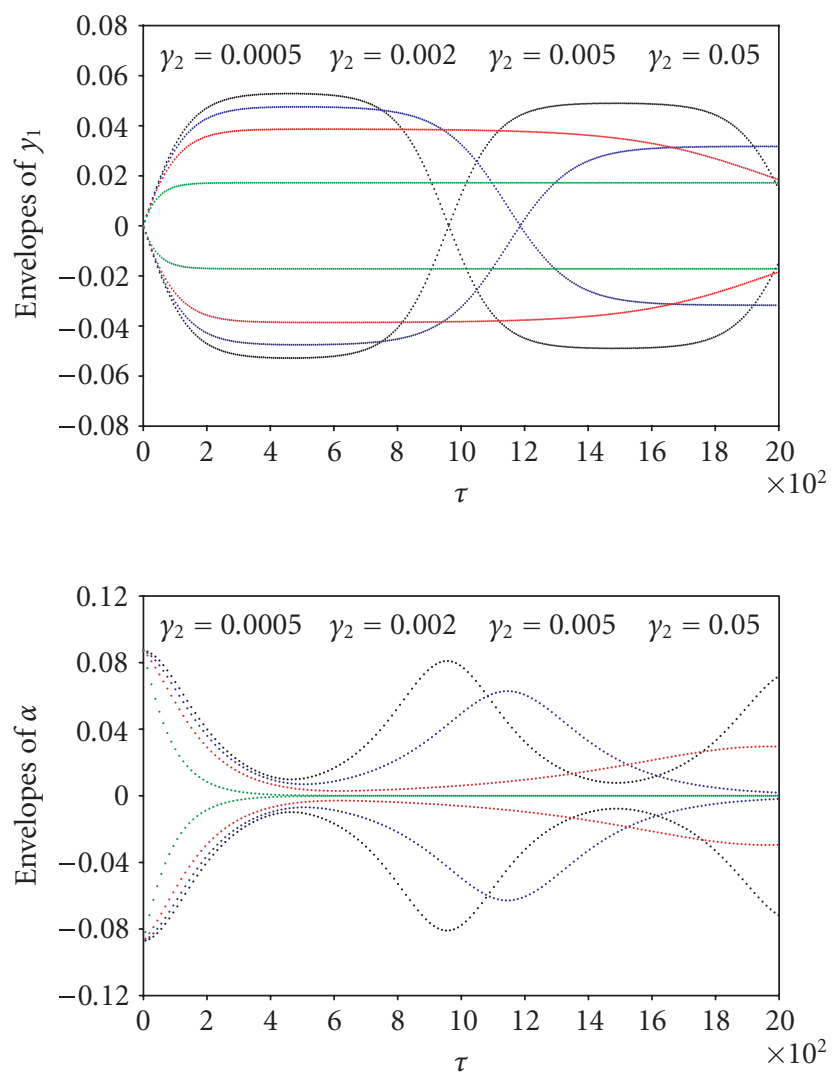

Figure 4.2. Influence of damping $\gamma_{2}$ on $y_{1}$ and $\alpha$ for $\beta=0.5, a_{1}=0, a_{2}=0.1, \gamma_{2}=\gamma_{3}=\gamma_{4}=0, b_{1}=0$.

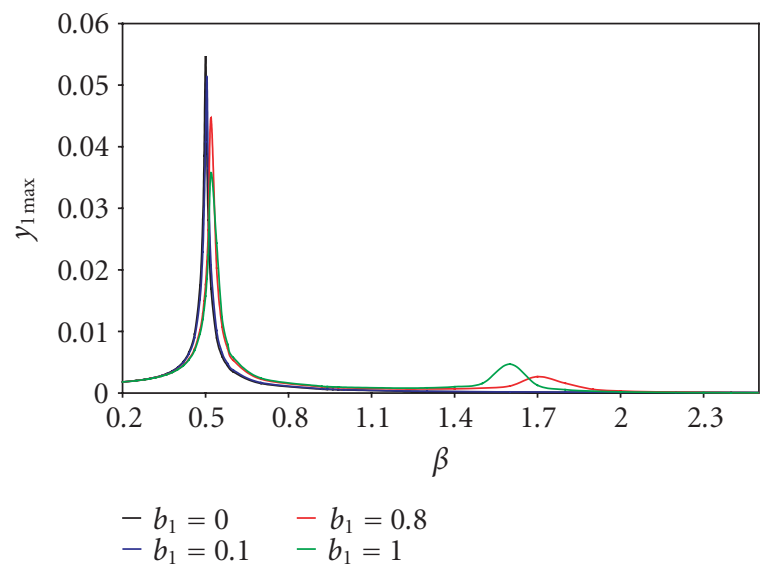

Figure 4.3. Internal resonance curves of $y_{1}$ for $\alpha(0)=5^{\circ}, a_{1}=0, a_{2}=0.1, \gamma_{1}=\gamma_{2}=\gamma_{3}=\gamma_{4}=0$. 

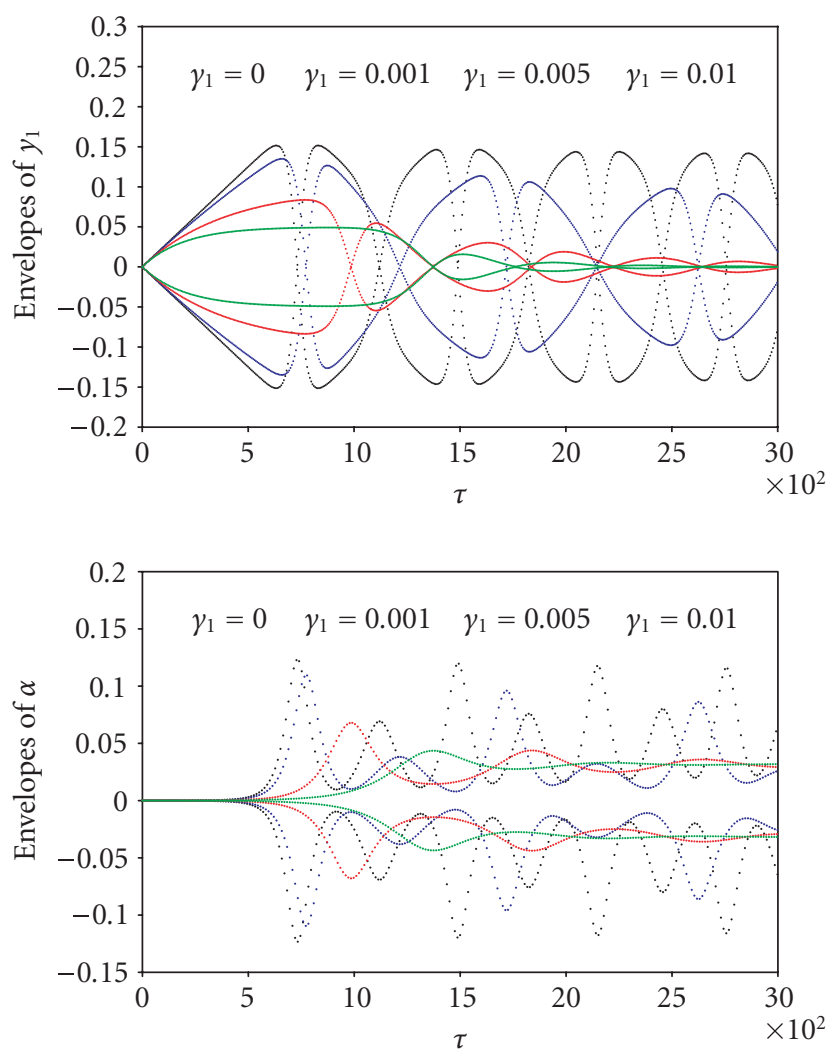

Figure 4.4. Influence of damping $\gamma_{1}$ on $y_{1}$ and $\alpha$ for $\beta=0.5, a_{1}=0.0005, a_{2}=0.5, \gamma_{2}=\gamma_{3}=\gamma_{4}=0$, $b_{1}=0$.

and $\alpha$, for control parameter $u_{1}=1.5$, are presented in Figure 4.11 for value of parameters: $u_{2}=1.5 ; q=0.2 ; \beta=0.5 ; a_{1}=0.01 ; a_{2}=0.1 ; \gamma_{1}=0.01 ; \gamma_{2}=\gamma_{4}=0, \gamma_{3}=2$, and $\gamma_{3}=20$, and in the internal and external resonances depending on selection Figure 4.12 for value of parameters: $u_{2}=1.5 ; q=0.2 ; \beta=0.5 ; a_{1}=0.01 ; a_{2}=0.1 ; \gamma_{2}=0.2 ; \gamma_{1}=\gamma_{3}=$ $\gamma_{4}=0$.

As can be seen from these diagrams, in this case when damping is put on the body of mass $M$ (Figure 4.11), the oscillations are transferred between modes of vibrations in infinite time, but in a case when damping is put on the pendulum (Figure 4.12) after time $\tau=1000$, steady state oscillations take place.

The resonant curves of the body of mass $M$ and of the pendulum for damping put on the body of mass $M$ and on the pendulum for the conditions of the main autoparametric internal resonance are shown in Figure 4.13.

Near of the physical system parameters, the amplitudes of vibrations of the coupled system may be related differently. The system presents some interesting nonlinear phenomena. Motions $y_{1}$ and $\alpha$ are periodic, multiperiodic, or quasi-periodic, but sometimes 
S. Danuta and K. Maciej 13
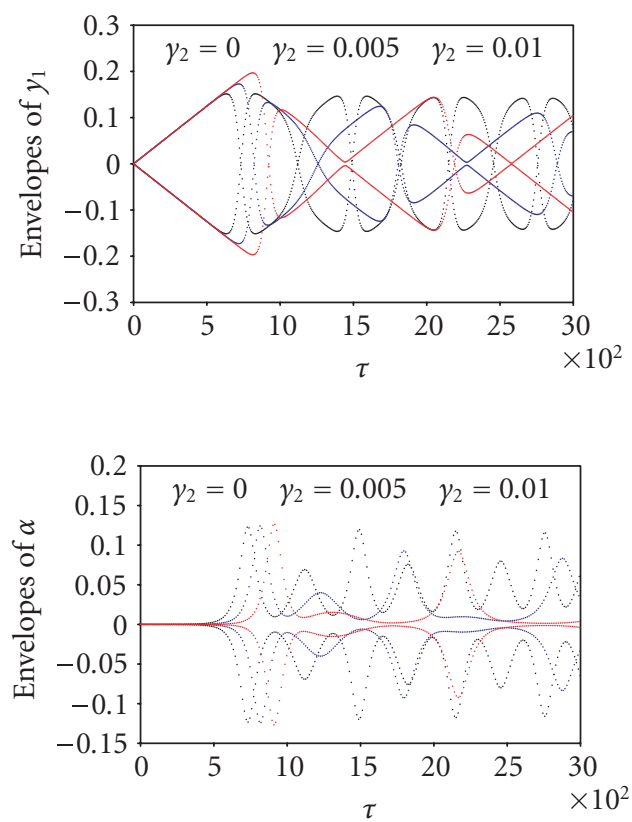

Figure 4.5. Influence of damping $\gamma_{2}$ on $y_{1}$ and $\alpha$ for $\beta=0.5, a_{1}=0.0005, a_{2}=0.5, \gamma_{2}=\gamma_{3}=\gamma_{4}=0$, $b_{1}=0$.
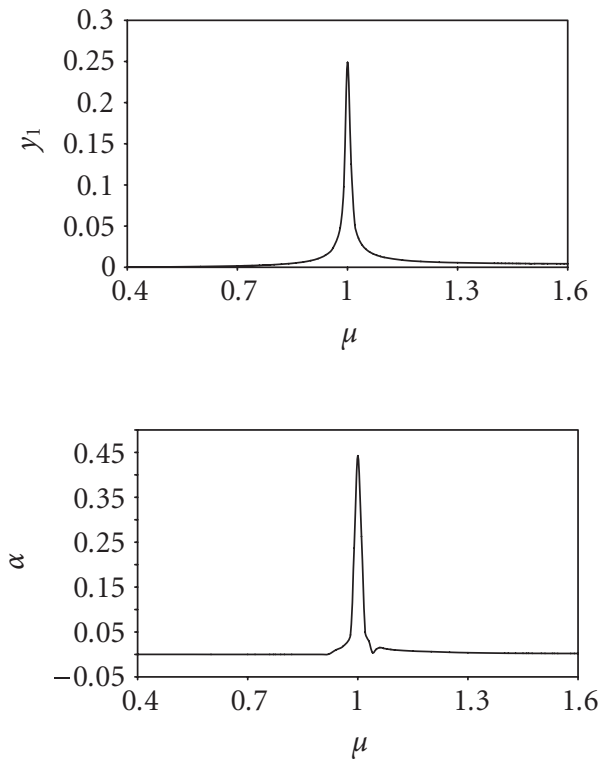

Figure 4.6. External resonance curves of $y_{1}$ and $\alpha$ for $\beta=0.5, a_{1}=0.001, a_{2}=0.1, \gamma_{1}=\gamma_{2}=\gamma_{3}=\gamma_{4}=$ $0, b_{1}=0$. 
14 Ideal and nonideal autoparametrical system
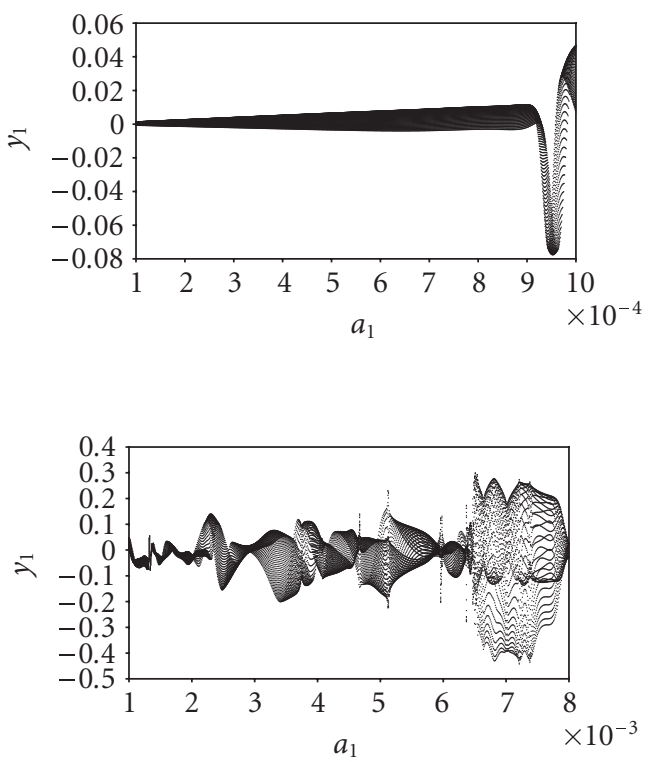

Figure 4.7. Bifurcation diagrams of $y_{1}$ for $\beta=0.5, a_{2}=0.3, b_{1}=0, \gamma_{1}=0.001, \gamma_{2}=0.002, \gamma_{3}=\gamma_{4}=0$, $\mu=1.01$.
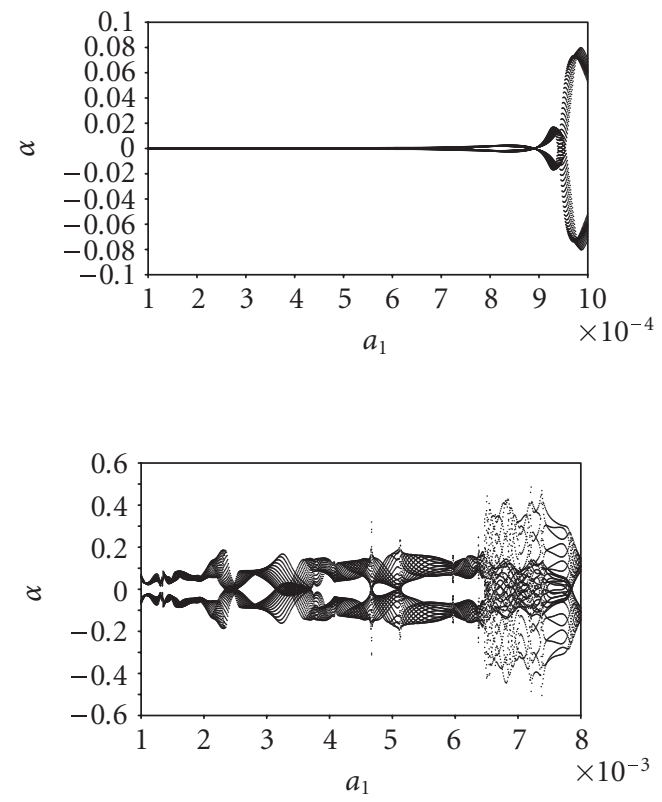

Figure 4.8. Bifurcation diagrams of $\alpha$ for $\beta=0.5, a_{2}=0.3, b_{1}=0, \gamma_{1}=0.001, \gamma_{2}=0.002, \gamma_{3}=\gamma_{4}=0$, $\mu=1.01$. 

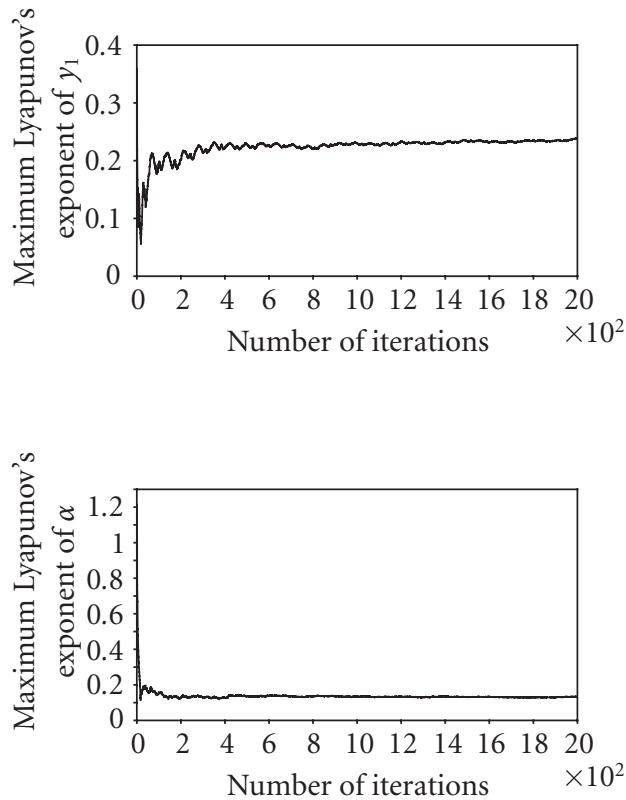

Figure 4.9. Maximal exponents of Lyapunov of $y_{1}$ and $\alpha$ for $a_{1}=0.007, \beta=0.5, a_{2}=0.3, b_{1}=0$, $\gamma_{1}=0.001, \gamma_{2}=0.002, \gamma_{3}=\gamma_{4}=0, \mu=1.01$.
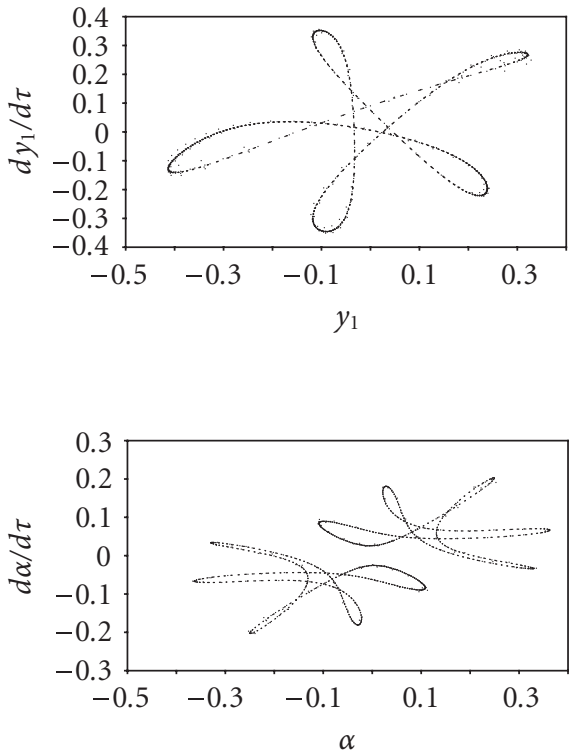

Figure 4.10. Poincaré maps of $d y_{1} / d \tau$ and $d \alpha / d \tau$ for $a_{1}=0.007, \beta=0.5, a_{2}=0.3, b_{1}=0, \gamma_{1}=0.001$, $\gamma_{2}=0.002, \gamma_{3}=\gamma_{4}=0, \mu=1.01$. 
16 Ideal and nonideal autoparametrical system
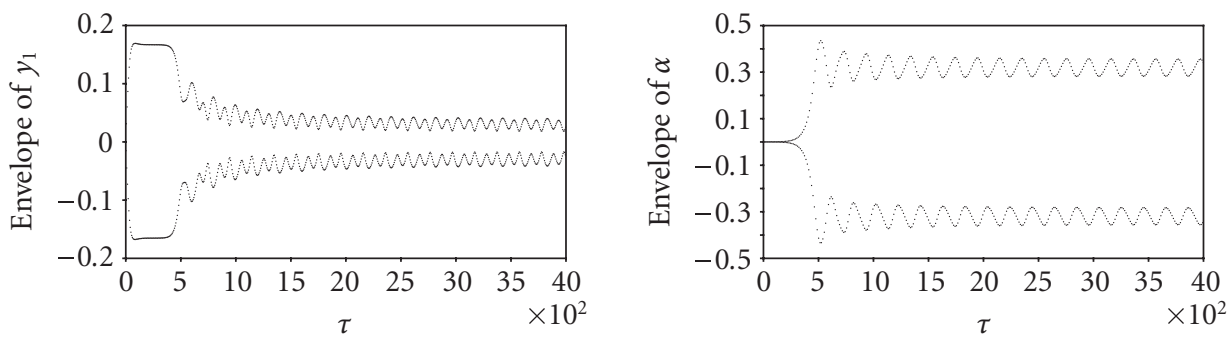

(a)
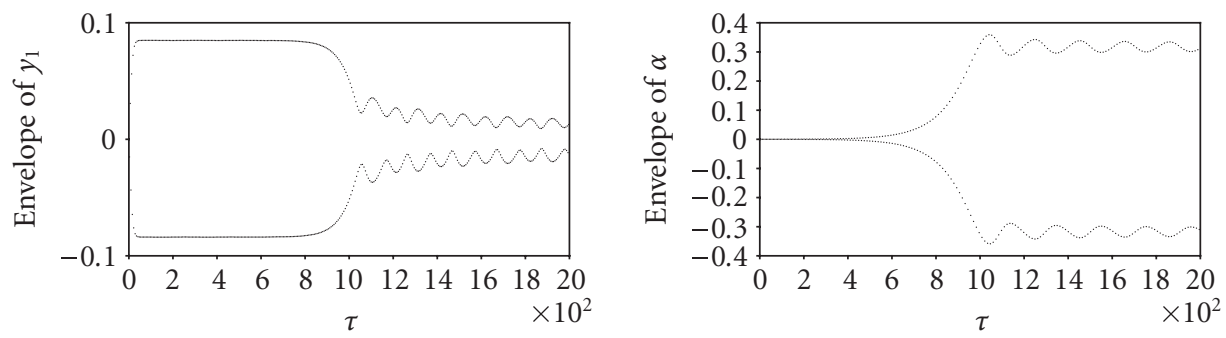

(b)

Figure 4.11. Influence of damping put on mass $M$ for $u_{1}=1.5, a_{1}=0.01, a_{2}=0.1, q=0.2, \gamma_{1}=0.01$ and $u_{2}=1.5, \gamma_{2}=\gamma_{4}=0$, (a) $\gamma_{3}=2$, (b) $\gamma_{3}=20$.
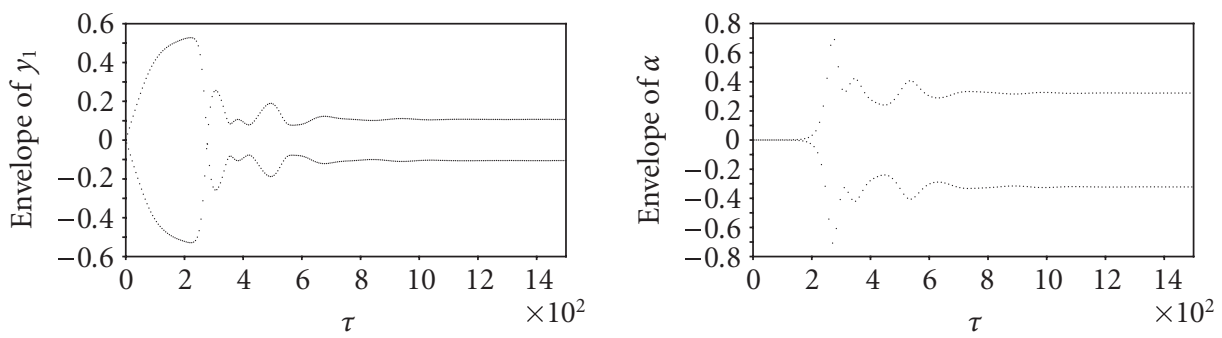

(a)
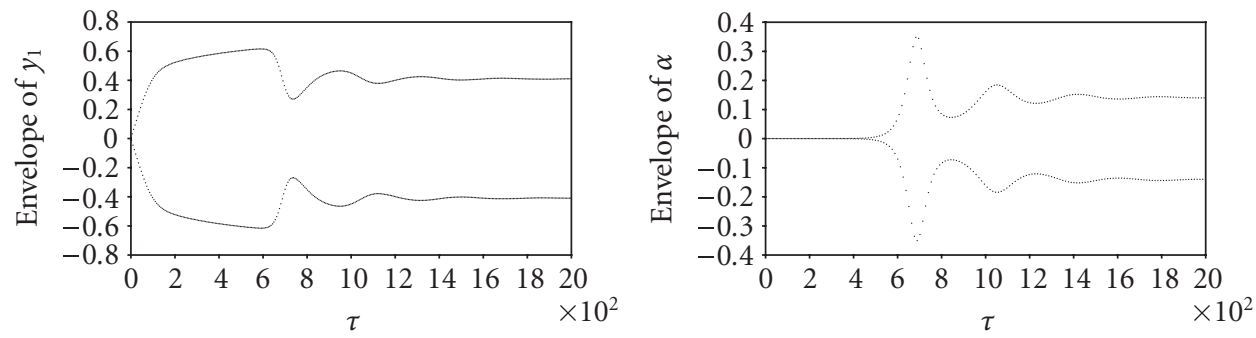

(b)

Figure 4.12. Influence of damping put on pendulum for $u_{1}=1.5, a_{1}=0.01, a_{2}=0.1, q=0.2, \gamma_{4}=0$ and $u_{2}=1.5, \gamma_{1}=\gamma_{3}=0$, (a) $\gamma_{2}=0.025$, (b) $\gamma_{2}=0.1$. 

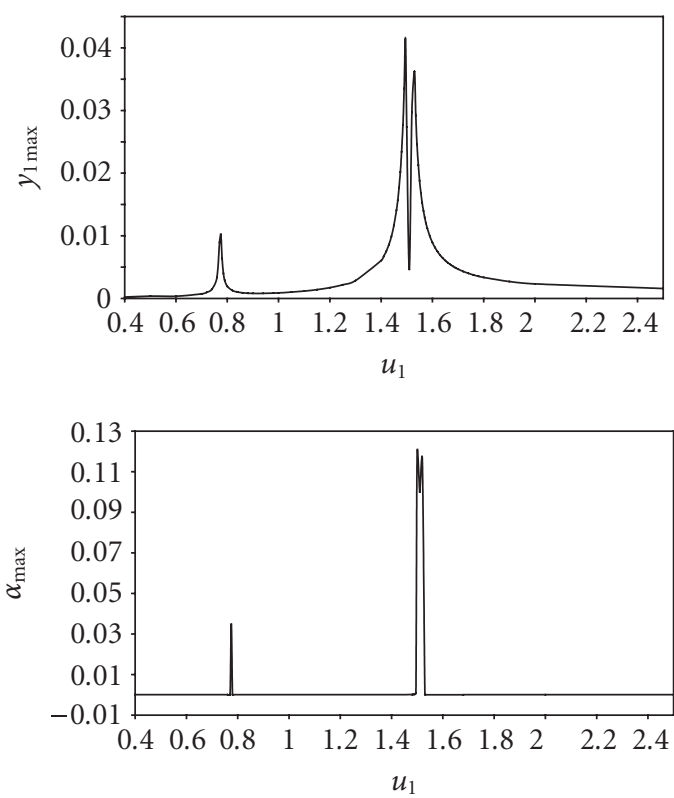

Figure 4.13. External resonance curves of $y_{1}$ and $\alpha$ for $a_{1}=0.001, a_{2}=0.1, q=0.2, u_{2}=1.5, \beta=0.5$, $\gamma_{1}=0.01$, and $\gamma_{2}=\gamma_{3}=\gamma_{4}=0$.
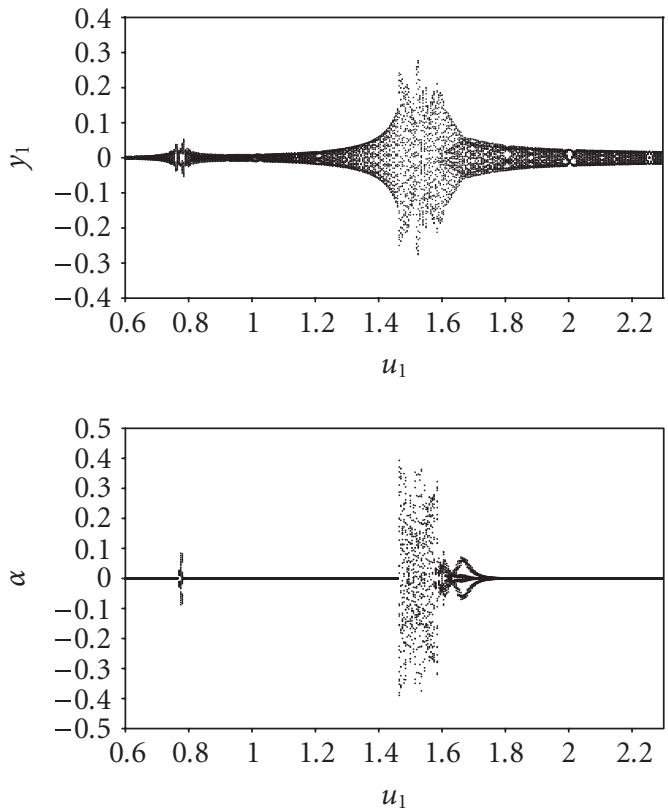

Figure 4.14. Bifurcation diagrams of $y_{1}$ and $\alpha$ for $a_{1}=0.01, a_{2}=0.3, u_{2}=1.5, q=0.2, \gamma_{1}=0.001$, $\gamma_{2}=0.002, \gamma_{3}=0.2, \gamma_{4}=0.02$. 

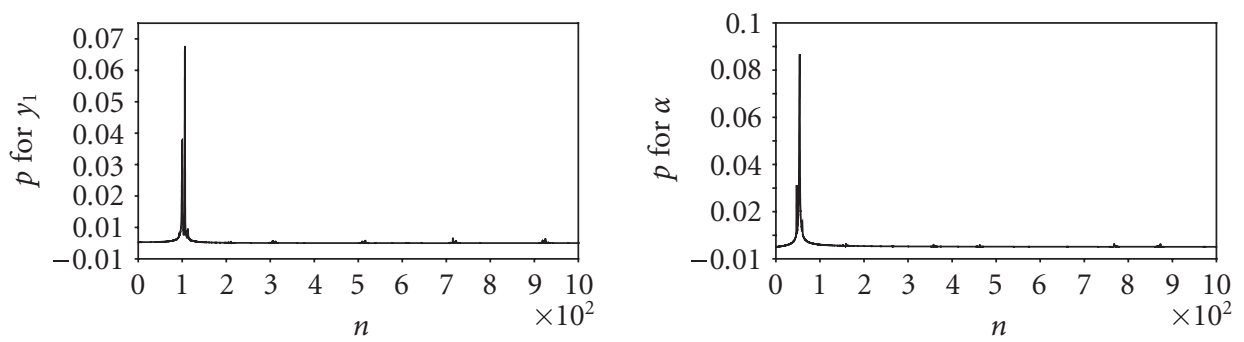

(a)
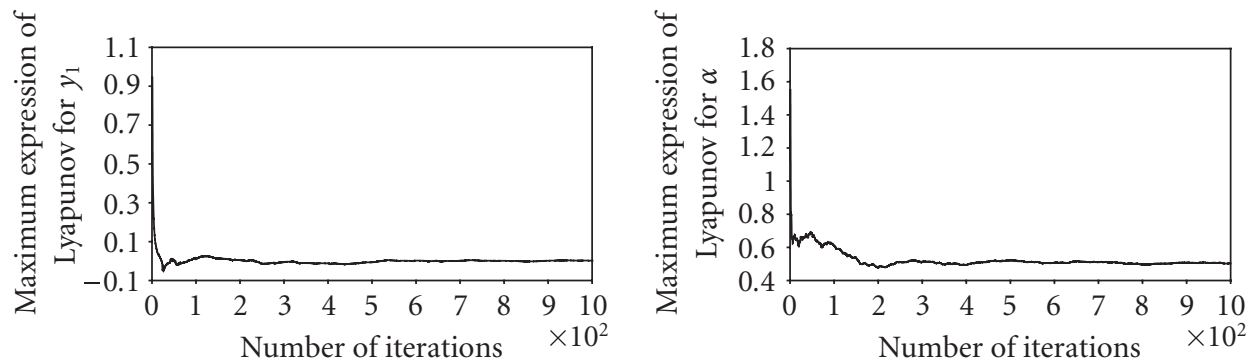

(b)
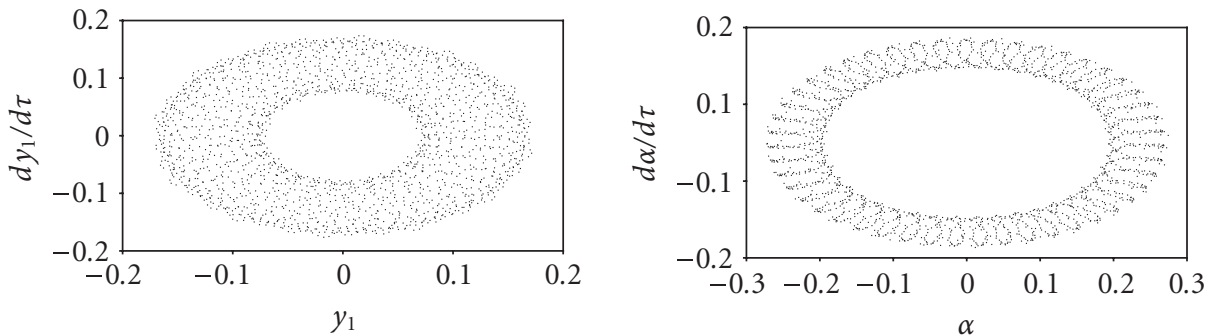

(c)

Figure 4.15. (a) FFT. (b) Maximum Lyapunov's exponent. (c) Poincaré maps of $y_{1}$ and $\alpha$ for $a_{1}=0.01$, $a_{2}=0.3, u_{2}=1.5, q=0.2, \gamma_{1}=0.001, \gamma_{2}=0.002, \gamma_{3}=0.2, \gamma_{4}=0.02$.

the motions of the body of mass $M$ and of the pendulum are chaotic. For characterizing, an irregular chaotic response forms a transition zone between one and another type of regular steady resonant motion, in the present paper, the bifurcation diagrams are constructed. Exemplary results for small nonlinear damping put on the body of mass $M$ and on the pendulum near internal resonance (near the principal autoparametric resonance for $\beta=0.5)$ that versus control parameter $u_{1}$ which depends on voltage are showed in Figure 4.14.

As it can be seen from these bifurcation diagrams, several phenomena can be observed, for example, existence of regular or chaotic attractors, and various bifurcations. To verify these phenomena, power spectral densities (their fast Fourier transform-FFT), Poincaré maps, and the largest Lyapunov exponents corresponding to coordinates $y_{1}$ and $\alpha$ are constructed. Exemplary results for control parameter $u_{1}=1.55$ are presented in Figure 4.15 for displacements $y_{1}$ and $\alpha$, respectively. 
These diagrams confirm the irregular (chaotic) nature of motion. The frequency spectrums are continuous, the Poincarè maps trace the strange attractors, and the largest Lyapunov exponents corresponding to coordinate $y_{1}$ and coordinate $\alpha$ are positive.

\section{Conclusions}

Motions of the nonlinear dynamical vibrating system with autoparametric coupling modeled as ideal or nonideal are analyzed in the paper. Several interesting phenomena were presented. The influence of linear and nonlinear damping parameters on energy transfer cycle has been observed.

For both models, except different kinds of periodic vibrations, also other kinds of irregular vibrations have been found. To construct the bifurcation diagrams for described models, we have to use different bifurcation parameters-for ideal model bifurcation parameter amplitude of excitation $a_{1}$ is used, but for nonideal model as control parameter parameter $u_{1}$ is used, which is related to the voltage of DC motor. When the bifurcation diagrams are plotted, several phenomena can be observed. All these phenomena have to be verified in the phase space. Nonperiodic attractors are traced by solving an initial value problem. The maximum Lyapunov exponents have been calculated in order to characterize the chaotic orbits.

\section{References}

[1] A. K. Bajaj, S. I. Chang, and J. M. Johnson, Amplitude modulated dynamics of a resonantly excited autoparametric two degree-of-freedom system, Nonlinear Dynamics 5 (1994), no. 4, 433-457.

[2] A. K. Bajaj and J. M. Johnson, Asymptotic techniques and complex dynamics in weakly nonlinear forced mechanical systems, International Journal of Non-Linear Mechanics 25 (1990), no. 2-3, 211-226.

[3] A. K. Bajaj and S. Tousi, Torus doublings and chaotic amplitude modulations in a two-degree-offreedom resonantly forced mechanical system, International Journal of Non-Linear Mechanics 25 (1990), no. 6, 625-641.

[4] G. L. Baker and J. P. Gollub, Chaotic dynamics: An Introduction, Cambridge University Press, Cambridge, 1996.

[5] J. M. Balthazar, D. T. Mook, H. I. Weber, R. M. L. R. F. Brasil, A. Fenili, D. Belato, and J. L. P. Felix, An overview on non-ideal vibrations, Meccanica 38 (2003), no. 6, 613-621.

[6] D. Belato, J. M. Balthazar, H. I. Weber, and D. T. Mook, On dynamical characteristics of the electromotor-pendulum, Nonlinear Dynamics, Chaos, Control and Their Applications to Engineering Sciences. Vol. 2: Vibrations with Measurements and Control (J. M. Balthazar, P. B. Gonçalvez, and J. Clayssen, eds.), vol. 2, Cruzeiro Editora e Artes Gráficas, Rio Claro, 1999, pp. 222-235.

[7] Chaotic vibrations of a nonideal electro-mechanical system, International Journal of Solids and Structures 38 (2001), no. 10-13, 1699-1706.

[8] K. L. Cavalca, I. L. E. Santos, and J. M. Balthazar, Analysis of combined effects of dry friction and non-linear restoring forces in rotors, Nonlinear Dynamics, Chaos, Control and Their Applications to Engineering Sciences. Vol. 2: Vibrations with Measurements and Control (J. M. Balthazar, P. B. Gonçalvez, and J. Clayssen, eds.), vol. 2, Cruzeiro Editora e Artes Gráficas, Rio Claro, 1999, pp. 206-221.

[9] R. M. Evan-Ivanowski, Resonance Oscillations in Mechanical Systems, Elsevier, Amsterdam, 1976.

[10] A. Fenili, J. M. Balthazar, and D. T. Mook, Some remarks on nonlinear and ideal or nonideal structure vibrating model, Proceedings of the 7th Conference on Dynamical Systems-Theory and 
Applications (J. Awrejcewicz, A. Owczarek, and J. Mrozowski, eds.), vol. 2, Łódź, 2003, pp. 549556.

[11] H. Hatwal, A. K. Mallik, and A. Ghosh, Forced nonlinear oscillations of an autoparametric system-part 2: chaotic responses, Transactions of the ASME: Journal of Applied Mechanics $\mathbf{5 0}$ (1983), 663-668.

[12] V. O. Kononenko, Vibrating Systems with Limited Power Supply, Illife Books, London, 1969.

[13] T. S. Krasnopol'skaya and A. Yu. Shvets, Chaotic interactions in a pendulum energy source system, Prikladnaya Mekhanika 26 (1990), no. 5, 90-96.

[14] F. C. Moon, Chaotic Vibrations, John Wiley \& Sons, New York, 1987.

[15] A. H. Nayfeh and D. T. Mook, Nonlinear Oscillations, John Wiley \& Sons, New York, 1979.

[16] L. Půst and O. Szöllös, Forced irregular oscillations of the two degrees of freedom nonlinear system, Proceedings of the 2nd European Nonlinear Oscillation Conference (EUROMECH '96), Prague, 1996, pp. 367-370.

[17] D. Sado, Periodic and chaotic oscillations of the autoparametric beam-pendulum system, Proceedings of the 3rd Biennal World Conference on Integrated Design and Process Technology (IDPT '98), vol. 6, Berlin, 1998, pp. 206-213.

[18] Chaos in autoparametric coupled mechanical systems, Proceedings of the 10th World Congress on the Theory of Machines and Mechanisms (IFToMM '99), vol. 4, Oulu, 1999, pp. $1638-1643$.

[19] _ Chaotic behavior of beam-pendulum system with dry friction, Proceedings of the 5th Engineering Systems Design and Analysis Conference (ESDA '00), vol. 82, Montreux, 2000, pp. $163-168$.

[20] , The chaotic phenomenon of a system with inertial coupling, Mechanics and Mechanical Engineering 6 (2002), no. 1, 31-42.

[21] Mechanical Engineering 7 (2004), 29-39.

[22] D. Sado and K. Gajos, Note on chaos in three degree of freedom dynamical system with double pendulum, Meccanica 38 (2003), no. 6, 719-729.

[23] D. Sado and M. Kot, The chaotic vibration of an autoparametrical system with non ideal source of power, Proceedings of the 7th Conference on Dynamical Systems-Theory and Applications (J. Awrejcewicz, A. Owczarek, and J. Mrozowski, eds.), vol. 2, Łódź, 2003, pp. 689-696.

[24] _ The periodic and chaotic vibration of an autoparametrical system with non ideal source of power, to appear in Journal of Computational Methods in Sciences and Engineering.

[25] M. Tsuchida, K. de Lolo Guilherme, J. M. Balthazar, G. N. Silva, and B. I. Cheshankov, On regular and irregular vibrations of a non-ideal system with two degrees of freedom. 1:1 resonance, Journal of Sound and Vibration 260 (2003), no. 5, 949-960.

[26] J. Warmiński, Regular and Chaotic Vibrations of Parametric and Self-Excited Systems with Ideal and Non-Ideal Energy Sources, Technical University of Lublin Press, Lublin, 2001.

[27] J. Warmiński, J. M. Balthazar, and R. M. L. R. F. Brasil, Vibrations of a non-ideal parametrically and self-excited model, Journal of Sound and Vibration 245 (2001), no. 2, 363-374.

Sado Danuta: Institute of Machine Design Fundamentals, Warsaw University of Technology, 84 Narbutta Street, 02-524 Warsaw, Poland

E-mail address: dsado@poczta.onet.pl

Kot Maciej: Institute of Machine Design Fundamentals, Warsaw University of Technology,

84 Narbutta Street, 02-524 Warsaw, Poland

E-mail address: maciej_kot@gazeta.pl 


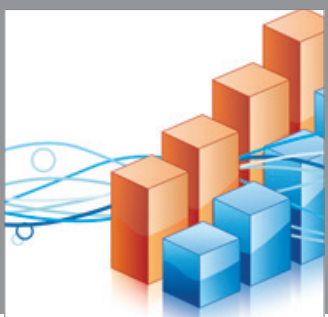

Advances in

Operations Research

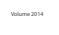

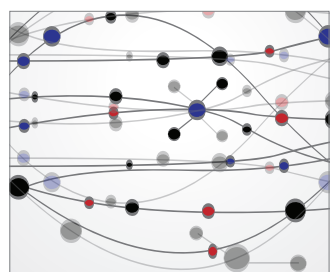

\section{The Scientific} World Journal
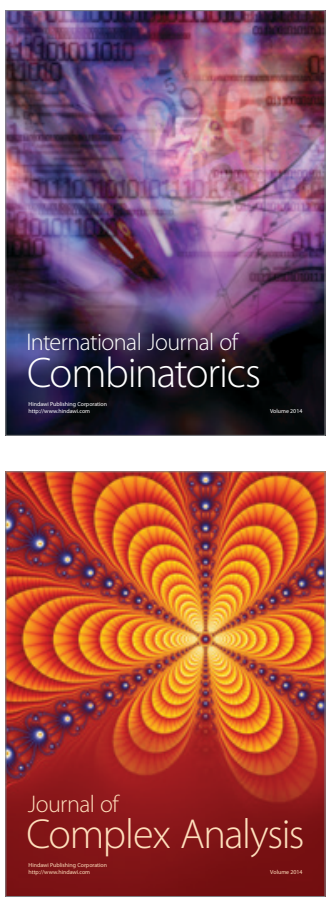

International Journal of

Mathematics and

Mathematical

Sciences
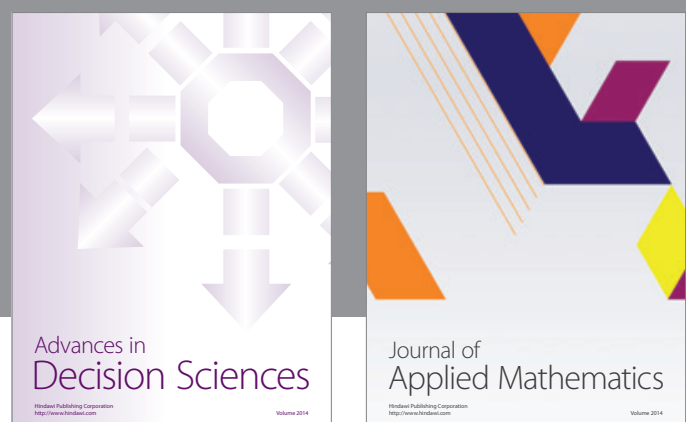

Journal of

Applied Mathematics
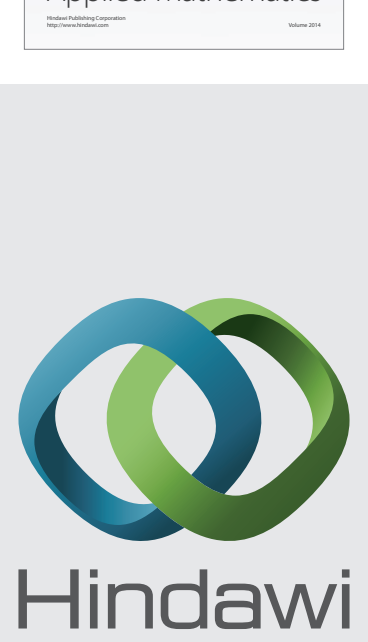

Submit your manuscripts at http://www.hindawi.com
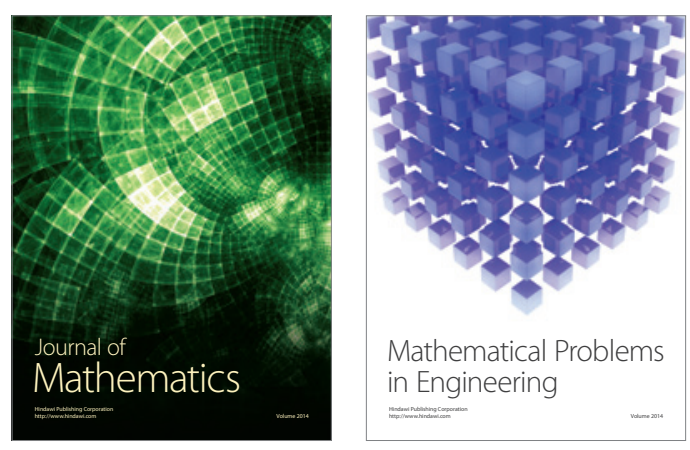

Mathematical Problems in Engineering
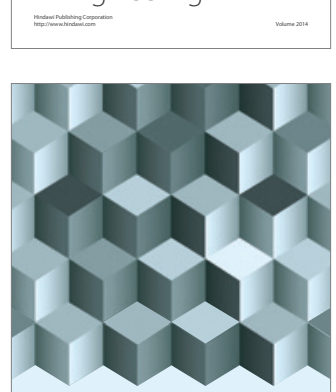

Journal of

Function Spaces
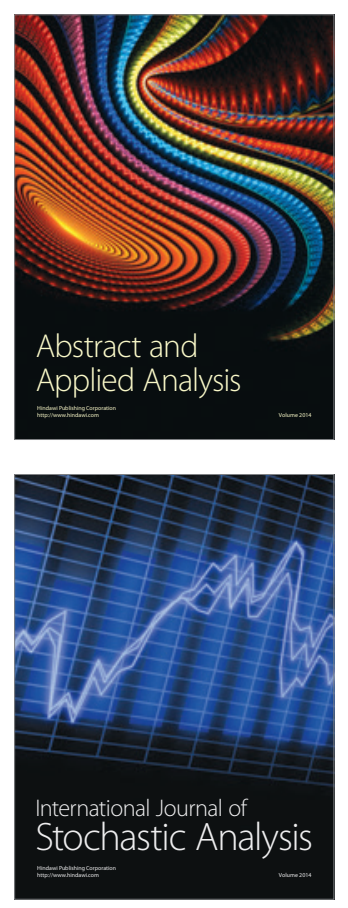

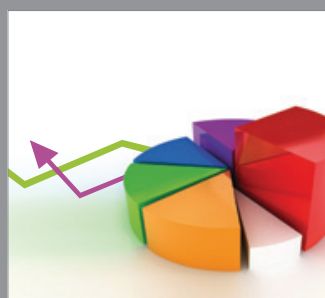

ournal of

Probability and Statistics

Promensencen
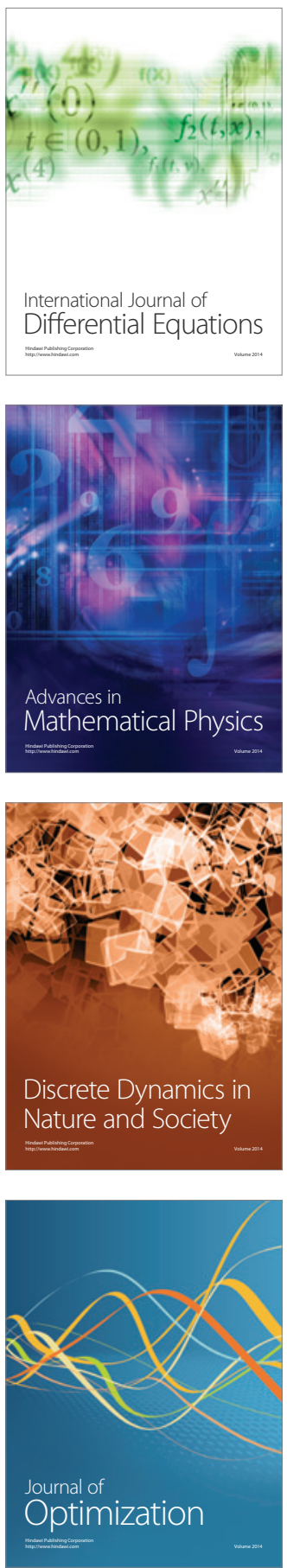